\title{
Le Sursis ou la dernière heure
}

Le Sursis or the Last Hour

Jean-François Louette

\section{CpenEdition \\ Journals}

Édition électronique

URL : https://journals.openedition.org/recherchestravaux/3360

DOI : $10.4000 /$ recherchestravaux.3360

ISSN : 1969-6434

\section{Éditeur}

UGA Éditions/Université Grenoble Alpes

\section{Édition imprimée}

ISBN : 978-2-37747-301-4

ISSN : 0151-1874

\section{Référence électronique}

Jean-François Louette, "Le Sursis ou la dernière heure », Recherches \& Travaux [En ligne], 98 | 2021, mis en ligne le 28 juin 2021, consulté le 09 août 2021. URL : http://journals.openedition.org/ recherchestravaux/3360 ; DOI : https://doi.org/10.4000/recherchestravaux.3360

Ce document a été généré automatiquement le 9 août 2021

(C) Recherches \& Travaux 


\title{
Le Sursis ou la dernière heure
}

\author{
Le Sursis or the Last Hour
}

Jean-François Louette

1 Du Sursis, deuxième volet de la trilogie romanesque de Sartre, Les Chemins de la liberté (1945), il ne s'agira pas ici d'évoquer l'ancrage autobiographique, et pas non plus les campagnes de rédaction, sur lesquelles au reste on a très peu de connaissances assurées - tout juste peut-on avancer que si le projet premier remonte à l'été 1938, le roman a été écrit du début 1942 à novembre 1944¹. On voudrait plutôt continuer à explorer, parmi les conditions de possibilité de ce texte, ce qui le rattache à la presse et au reportage ${ }^{2}$. Tant il est vrai qu'un beau jour le roman s'est trouvé saisi par l'information ${ }^{3}$ : en France, sans doute vers 1830 ; en Angleterre, probablement plus tôt - songeons aux romans de Defoe. Il se peut qu'il ne s'en soit depuis lors jamais dégagé, mais non sans rébellion. Par exemple : qui, du journaliste ou du romancier, racontera le mieux la dernière heure?

\section{La presse}

2 Le Sursis relate la crise dite de Munich. Cette crise, Sartre l'a bien évidemment lui-même vécue. Dans une longue lettre - de septembre 1938, précisément - à Simone de Beauvoir, il analyse en détails la situation et conclut ainsi : « [...] au cas, même, où la guerre serait évitée, ça ne fait ni très beau ni très gai. Tout de même les gens sont plus calmes ici [à Paris], plus heureux : il leur semble qu'ils vont peut-être avoir un sursis de quelques années. Pour moi je n'en demande pas plus en cet instant ${ }^{4}$. » Ainsi s'annonce le titre Le Sursis (et aussi l'importance de l'instant). Mais le 31 décembre 1939, Sartre soumet une autre hypothèse à Beauvoir (qui n'a pas dû être convaincue) : le roman qui fera suite à L'Âge de raison doit s'intituler "Septembre ${ }^{5}$ ». Le 2 janvier 1940, Sartre, mobilisé en Alsace, écrit à son épouse morganatique: «Quand je serai à Paris, je prendrai tous les Paris-Soir de septembre 38 pour ma documentation ${ }^{6}$. »

3 Par quoi il suit lui-même le conseil qu'il avait donné un bon mois plus tôt à Beauvoir. Lorsqu'elle avait décidé de situer l'intrigue de son propre roman, L'Invitée, pour partie 
en 1938, elle avait demandé à Sartre « un historique de l'an dernier »; réponse : «Vous feriez beaucoup mieux d'aller à la Bibliothèque nationale consulter les journaux, ça vous donnerait l'atmosphère ${ }^{7}$. » Et trois jours plus tard, le 21 novembre 1939 : «Lisez des journaux, ma petite fleur, tous les journaux de l'année pour vous y reconnaître et trouver un écho des discussions d'alors ${ }^{8}$.» Celles, par exemple, que Robert de SaintJean, l'amant en titre de Julien Green, suivait à Londres pour Paris-Soir.

Le roman existentialiste se fonde, entre autres, sur les informations que les journaux procurent aux petites fleurs présentes et aux grands écrivains futurs. Sartre connaît bien la presse de son époque. Il lui arrive d'aider telle de ses amours contingentes à se repérer dans ce champ; ainsi, s'adressant à Louise Védrine (c'est-à-dire Bianca Bienenfeld, cousine de Georges Perec), à la veille de la guerre : «Dieu sait de quels journaux vous disposez à Annecy. Ne lis surtout pas L'Intransigeant qui est l'organe du munichois Chautemps. Lis L'Euvre, le Paris-Soir et Le Temps'. » Il conseille donc, en un beau geste non partisan, une feuille radicale socialiste, un organe centriste, et la gazette officieuse du Quai d'Orsay, très à droite en politique intérieure et violemment anti-communiste.

\section{L'histoire immédiate}

$5 \quad$ Pour écrire Le Sursis et L'Invitée, Sartre et Beauvoir lisent donc les journaux de l'année 1938. Mais ce premier type de textualisation de l'événement ne satisfait pas l'exigeante Simone. Le 16 décembre 1939, elle écrit à Sartre : «Ah ! pour mon roman» - toujours L'Invitée - je vais lire Fabre-Luce, mais je n'ai pas du tout l'intention de brosser de grands tableaux historiques; je veux juste que les événements soient replacés ${ }^{10}$ ». Quatre jours plus tard, indiquant ses scrupules et son désir d'exactitude : «j'ai acheté le Fabre-Luce pour avoir ma conscience avec moi ${ }^{11}$. » Le 29 décembre, la voici à Megève, attendant son professeur de ski : "J'avais emporté un livre dans ma ceinture, l'histoire de Munich par Fabre-Luce et je l'ai lu ${ }^{12}$.» Pas de temps perdu à rêvasser au bord des pistes.

6 Alfred Fabre-Luce avait publié chez Grasset une Histoire secrète de la conciliation de Munich, en 105 pages, avec un achevé d'imprimer du 21 octobre 1938: même pas un mois après le dénouement de la crise. Tel est l'opuscule qui va skier avec Beauvoir. Grand bourgeois, époux de la princesse Charlotte de Faucigny-Lucinge (une descendante de Charles $\mathrm{X}$ ), très hostile au Front populaire, proche du PPF de Jacques Doriot, Fabre-Luce critique le «centralisme despotique " des Tchèques qui oppriment les pauvres minorités allemandes des Sudètes, et il s'en prend à "l'internationale belliciste ${ }^{13}$ », qu'on devine rouge.

7 Il s'agit d'un effort d'histoire immédiate. "Que dira l'Histoire? ", demande l'auteur, et à la page suivante, avec l'humilité coutumière aux historiens, il répond: «La vérité, c'est que... », se faisant fort de porter un «jugement équitable ${ }^{14}$ ». Mais, outre le titre "vendeur ", pour ne pas dire racoleur, la forme de son texte est très significative: après un prologue et une présentation des "quatre joueurs de poker" (Hitler, Mussolini, le parti de la guerre et le parti de la paix en France et en Angleterre), chaque chapitre porte pour sous-titre une date, du " 12 septembre ", au " 29-30 septembre ». L'ordre est chronologique, et cette plaquette historique se propose donc comme une chronique - rédigée au présent. 
Histoire tellement immédiate que la frontière qui la sépare du reportage semble bien mince. Et peut-être Fabre-Luce a-t-il utilisé des articles de sa propre main, écrits pour tel ou tel journal. Cela pourrait se vérifier, mais inutile d'accorder trop de temps à Fabre-Luce - croyons-en Sartre répondant à Beauvoir, le 26 mai 1940 : « Vous m'avez grandement surpris en énumérant les livres que vous allez m'envoyer. J'ai demandé un Fabre-Luce, moi ? Il faut que je sois fou. Je tiens les 2 Fabre-Luce, pour des cons ${ }^{15}$. »

Je ne connais point d'indice montrant, même si la chose n'a rien d'impossible, que Sartre ou Beauvoir ait lu l'opuscule de Daniel Halévy, lui aussi publié chez Grasset, intitulé 1938, une année d'histoire, et qui porte comme achevé d'imprimer le 29 décembre 1938. En revanche, Sartre s'est beaucoup appuyé sur la Chronique de septembre de son ami Nizan, rédacteur en chef du quotidien communiste Ce soir, chargé de la politique étrangère : non seulement sur les faits qu'il recense ou établit, mais aussi - je l'ai montré ailleurs - sur sa très intéressante "Introduction", qui compare les démarches du journaliste et celles de l'historien ${ }^{16}$. Nizan se définit, en tant que "rédacteur diplomatique ", comme un "historien de l'immédiat », et l'ouvrage est imprimé pour Gallimard le 20 mars 1939 - donc six mois après la crise de Munich ${ }^{17}$. Deux remarques ici.

D'une part, Sartre reprend les conclusions politiques de Nizan dans Le Sursis, comme les résument ces lignes du carnet XIV, qui par avance, le 8 mars 1940, peignent Jacques Delarue, grand bourgeois français, le frère de Mathieu, l'un des personnages principaux du Sursis : "C'est la bourgeoisie qui a empêché la guerre en 38 et décidé la capitulation de Munich, plus encore par peur de la victoire que de la défaite. Elle redoutait que la guerre ne profitât au communisme. [...] La guerre en 38 pouvait être l'occasion d'une révolution ${ }^{18} »-$ cette révolution dont rêve un personnage absent de L'Âge de raison mais qui apparaît dans Le Sursis, l'ouvrier communiste de Saint-Ouen, Maurice.

11 D'autre part, Sartre a médité sur ce que j'appellerais le pari de Nizan : durant la crise de Munich, pour le journaliste, la précipitation régnait, note Nizan, «l'heure des éditions commandait ; le public impatient de savoir si la guerre ou la paix l'emportait, attendait les nouvelles comme du pain »; on comprenait "qu'il fallait risquer, et décider pour une version qu'on savait sans doute au moins prématurée "; de façon générale, tel est «l'esprit de risque et de pari » que suppose le métier de rédacteur diplomatique, ou de journaliste de l'immédiat, avec le péril d'être démenti par l'événement ${ }^{19}$. Toutes ces remarques serviront à Sartre de matière pour un épisode brillant de son roman, qui montre, le 26 septembre 1938, un journaliste communiste anonyme, lequel, sur un mot ambigu de Gamelin revenant de Londres, croit que cette fois la guerre va éclater et file l'annoncer à L'Huma : écoutant trop ses désirs, il parie - et il perd.

\section{Distance romanesque et roman d'actualité}

Nizan a fourni à Sartre une autre donnée importante : l'exemple d'un roman d'actualité à implications politiques, troisième forme de textualisation de l'événement historique. En 1939, dans un article intitulé «Ambition du roman moderne ", Nizan évoque l'idée de « distance romanesque » $:$ la distance « entre l'événement que le romancier décrit et le moment où le romancier écrit ${ }^{20} »$. Notion qu'avait développée un critique marxiste, Pierre Abraham - collaborateur, notamment, de la revue Europe -, dans Créatures chez Balzac (Gallimard, 1931). Mais alors qu'Abraham plaide pour une distance minimale 
d'une quinzaine d'années, Nizan tient que cette distance peut être très mince, voire quasi inexistante, et il oppose les romans de la fuite hors du monde (à la Giono, dit-il), et les romans qui affrontent la réalité contemporaine, l'actualité. C'est là défendre son propre roman, Le Cheval de Troie, qui relate une grève violente à Villefranche, en juin 1934, est écrit en 1934-1935, et paraît chez Gallimard en octobre 1935.

13 Nizan n'est évidemment pas le seul, dans les années trente, à pratiquer le roman d'actualité. On peut songer à deux autres écrivains dont Sartre suivait de près la production. D'abord, André Chamson: L'Année des vaincus évoque l'année 1933, comme moment d'un basculement sans retour dans les relations entre la France et l'Allemagne, et d'ailleurs aussi dans l'évolution personnelle de l'auteur, qui passe du pacifisme à l'antifascisme ; le roman, écrit en 1933-1934, pré-publié d'août à novembre 1934 dans La NRF, paraît chez Grasset avec un achevé d'imprimer du 8 novembre 1934. Du même auteur, La Galère, qui transpose le 6 février 1934, paraît avec un a. i. chez Gallimard le 6 février 1939, exactement cinq ans plus tard. Souvenons-nous que Sartre proposera à Chamson d'entrer dans l'équipe fondatrice des Temps modernes - peut-être vexé par son refus, il l'exécutera ensuite dans le dernier chapitre de Qu'est-ce que la littérature?

Et, bien sûr, il y a, quasiment hors concours, le prestigieux exemple d'André Malraux. L'Espoir, qui relate des événements survenus de juillet 1936 à mars 1937, paraît chez Gallimard à la fin de 1937, alors que la guerre d'Espagne n'est pas encore achevée. Malraux impose une nouvelle figure dans le champ littéraire, qu'on peut ainsi dessiner - ou caricaturer? Désormais « le grand écrivain [...] incarne l'hypostase trinitaire de l'artiste du style, de l'instituteur républicain et du grand reporter $^{21} »$. Telle sera à peu près la figure de Sartre à l'automne de 1945, l'instituteur étant devenu agrégé, et le reporter ayant pour titres de qualification les reportages sur la Libération de Paris écrits à la demande de Camus pour Combat - et, aussi, comme nous venons de le voir, Le Sursis, quoique de façon moins manifeste. Croyons-en Bernard Frank, qui prête à Sartre cette formule, dans une conversation à propos des Chemins de la liberté : « Le rêve, maintenant, c'est, suivant la formule de Malraux, d'introduire le reportage dans l'imaginaire ${ }^{22}$."

\section{Roman et reportage, trois variations}

Dans son récit autobiographique, traduit en français par Vladimir Pozner en 1926, Victor Chklovski, grande figure du formalisme russe, résume ainsi l'une de ses thèses principales : «À sa source l'art est ironique et destructif. [...] Il crée de nouvelles formes en canonisant les formes subalternes ${ }^{23}$. " C'est ce qui se passe pour tout un pan du roman français dans les années vingt et trente: en cet âge d'or de l'édition, il se renouvelle en se nourrissant du grand reportage ${ }^{24}$. Encore faut-il distinguer, au sein de l'ensemble générique du "roman-reportage", des variations ou des nuances: le roman-reportage proprement dit (Dreiser, modèle rejeté par Sartre) n'est pas le roman à allure de reportage (Malraux, modèle évident et contesté), qui n'est pas le reportage à allure de roman (Pozner, modèle partiel et caché).

Dans une conférence au Havre, sur Dos Passos, le 6 février 1933, Sartre mentionne un exemple, et un seul, de « roman-reportage »: Une tragédie américaine de Dreiser ${ }^{25}$. De ce texte, malgré ses qualités satiriques - roman athée, roman anticapitaliste -, Sartre n'a pas une très haute idée. Moins bon que du Dos Passos, juge le conférencier: Dreiser désindividualiserait ses personnages à l'excès - de fait le romancier américain veut 
montrer qu'aucun ou presque ne s'arrache à la banalité des clichés et des codes sociaux. Ajoutons, même si cela ne fut point noté par Sartre, que le narrateur d'Une tragédie américaine est trop présent et trop savant pour ne pas évoquer fâcheusement la figure de l'auteur-Dieu que Sartre en février 1939 reprochera à Mauriac d'introduire en ses romans.

Quant au roman à allure de reportage, le modèle ici pour Sartre est L'Espoir. Non seulement la distance romanesque y est très mince, mais de plus - bref rappel Malraux s'inspire, entre autres, des reportages de Louis Delaprée pour Paris-Soir, republiés de façon posthume dans Mort en Espagne, en février 1937. L'Espoir a été prépublié dans Ce soir en trente-deux épisodes, du 3 novembre au 7 décembre 1937, la rédaction insérant « de nombreuses photos qui accompagnent étroitement le texte ${ }^{26}$ ». Le roman met en scène plusieurs figures de reporters, dont celle, frappante, d'un Américain, Shade, qui dicte un article sous le bombardement de Madrid par les franquistes. On a pu soutenir que la discontinuité du texte - dans la segmentation abondante (parties, chapitres, etc.) et dans le style - évoque les pratiques de l'écriture journalistique; et que la technique des foyers de perception (ou focalisation interne) transpose - je vais vite - le cadrage subjectif (limité et partiel) propre au reportage.

Dans Le Sursis Sartre ne cesse de dialoguer avec L'Espoir, sur divers modes. Celui de l'allusion : l'un des personnages, Gomez, fait la guerre en Espagne, et au moment de le rencontrer à Juan-les-Pins son ami Mathieu Delarue pense à l'infériorité des républicains en armement - «des dinamiteros contre des tanks»-, ce qui évoque une scène célèbre du roman de Malraux, tout comme la mention du même Gomez se battant à la Cité Universitaire de Madrid ${ }^{27}$. Mais aussi le mode de la concurrence, voire du reproche : Sartre écrit en effet une scène de guerre en Espagne, la mort d'un franquiste, un Marocain, tué par un Belge ; scène en trois moments, au cœur de laquelle est adopté le point de vue du soldat de Franco - ce que Malraux ne fait jamais dans L'Espoir ${ }^{28}$. Enfin, le mode du clin d'œil: un discours haineux de Hitler suscite en Gomez, qui l'écoute à Barcelone, et qui a dit à Mathieu lors de leur dîner que la guerre en Espagne était perdue, " un sentiment depuis longtemps enseveli, quelque chose qui ressemblait à de l'espoir ${ }^{29}$ ». Voilà qui s'appelle signer une relation intertextuelle... (Même si l'on revoit rapidement Gomez plus loin, et que son dernier mot, plus sartrien, sera alors « Les salauds ».)

19 Trois différences majeures néanmoins entre Le Sursis et L'Espoir (outre le fait que la crise de Munich n'est pas la guerre d'Espagne, etc.). Dans une lettre à Beauvoir du 29 novembre 1939, Sartre évoque "cette grande machine à fusées qu'est L'Espoir ${ }^{30}$ ». Pour matière, une humanité largement héroïque. Pour manière, plus d'une fois une emphase presque épique - ce qui notamment séparait le roman de Malraux du reportage stricto sensu. Sartre avait un temps pensé donner pour titre aux Chemins de la liberté «La Grandeur ${ }^{31}$ ». Il y renoncera, prenant ainsi ses distances avec l'univers de Malraux ; il ne s'agissait d'ailleurs pas de la même grandeur : non pas les fusées, mais l'authenticité. Aussi Sartre peint-il la grandeur chez des personnages sinon infirmes - quoique les amours de Charles et Catherine, atteints du mal de Pott, montrent la grandeur dans l'abjection -, du moins ordinaires. En 1945, Henri Thomas, poète et romancier que publie déjà Gallimard, admire "Gros-Louis, l'illettré (la source des réflexions sur soi se trouve ainsi fermée), le naïf, que définissent les coups infligés par le monde extérieur. Sa modeste épopée à l'intérieur du Sursis est admirablement contée; il y a là une note entièrement nouvelle chez Sartre, faite de bonhomie et 
d'humour fraternel ${ }^{32}$.» Bernard Frank notera un sentiment analogue à propos du Sursis tout entier; l'événement mondial y est certes roi, mais les personnages ont une présence, «les consciences existent, même si elles existent en tant que consciencesnoyées-broyées par l'événement. Il y a une bonne humeur du désespoir, une bonne humeur du talent ${ }^{33}$. " Tragédie, mais de bonne humeur, épopée, mais modeste : donc un ton moins crispé et moins tendu vers les sommets que ne l'est celui de L'Espoir, ton que Sartre cherche à produire au plus juste dans le souvenir de Pascal (misère et grandeur), de Hugo (le sublime et le bouffon), peut-être aussi de Queneau.

L'enjeu profond - deuxième différence - du refus des fusées à la Malraux serait la conception et la figuration du peuple ou de la nation en guerre. Phrase décisive de Sartre dans le carnet XIV, notée le 6 mars 1940 : une " nation armée démocratique qui prend conscience de soi comme telle, je prétends qu'elle est aux antipodes de l'héroïsme », fût-ce, ajouterais-je, celui du peuple espagnol ; la guerre devient plutôt "un service civil», "une corvée qui incombe à tous»-qu'on supporte avec un " humanisme bonhomme " (c'est bien la modeste épopée); et "il faut s'en réjouir », ajoute Sartre, "parce que ça aussi, ça contribue à tuer la guerre ${ }^{34}$ ». En Sartre, ici, le pacifisme mesuré d'Alain critique le bellicisme exalté de Malraux.

Enfin, si Malraux et Sartre ont tous deux écrit des romans de montage, ils ne pratiquent pas le même type de montage, et Sartre est allé plus loin que son prédécesseur sur cette voie. Je m'explique, mais il faut d'abord définir rapidement la notion de littérature de montage $e^{35}$.

En France, à ma connaissance, elle se dessine en 1929 sous la plume de Vladimir Pozner dans son Panorama de la littérature russe contemporaine; deux pages, dans la conclusion, portent sur « la question du montage d'œuvres russes actuelles. Ce terme cinégraphique [sic] s'applique exactement à la plupart des livres parus en Russie et que, d'ailleurs, le cinéma a influencés ${ }^{36} »$. Puis, en juillet 1932, dans un article de Monde (la revue hebdomadaire fondée par Barbusse), article intitulé «Une nouvelle technique du roman ", René Lelu, pour la première fois en France, semble-t-il, emploie l'expression "roman-montage ", à partir et à propos d'une seule œuvre, $42^{\text {nd }}$ Parallel de John Dos Passos, dont la traduction française paraitra en 1933. En juin 1934, dans un compte rendu pour La NRF, Malraux note que, pour parler « en termes de cinéma ", à côté d'une « littérature de photographies commence à se constituer une littérature de montage »: le roman Les Traqués de Michel Matveev est ainsi une "suite de tableaux coordonnés par une fatalité ", un récit qui "ignore le temps » et "aboutit non à une durée mais à des scènes ${ }^{37}$ »; 1934 est l'année où Malraux forme le projet d'adapter au cinéma La Condition humaine avec Eisenstein. Mais retournons à Pozner, que Lelu et Malraux ont peut-être lu: la définition qu'il avance repose sur trois critères (qui d'évidence s'appliquent à L'Espoir et au Sursis).

Un roman de montage ne se comprend pas aisément à la première lecture : il produit un trouble, une désorientation. Un roman de montage pratique l'insertion de documents bruts : « lettre, télégramme, journal intime, décret, affiche, etc. sont considérés comme œuvres d'art», et donc "insérés tels quels dans les livres ${ }^{38}$ ". Enfin, un roman de montage suppose l'abandon du « récit suivi» (chronologique, s'ordonnant en gros selon la vie d'un seul personnage principal), et donc la disjonction de la trame narrative en «morceaux séparés »; ainsi, ajoute Pozner, dans L'Année nue de Boris Pilniak. Roman traduit en 1926 dans une collection de Gallimard - « Les Jeunes Russes » - que Sartre et Beauvoir ont fréquentée, indique cette dernière dans La Force de l'âge; et de Pilniak, 
écrivain auquel Brunet pense dans Le Sursis, ils avaient au moins lu avec grand intérêt La Volga se jette dans la Caspienne ${ }^{39}$.

On pourrait préciser un peu : la disjonction ou séparation s'accompagne de l'effacement de l'auteur, et elle implique la pluralité des fils narratifs, ou pluri-linéarité. On le voit tout de suite, la disjonction ou la séparation ne peuvent malgré tout aller sans la juxtaposition; c'est elle, comme l'a dit Eisenstein, qui crée des effets de montage, et permet l'analogie entre roman et cinéma. En 1945, Sartre écrit dans sa «Prière d'insérer » que Le Sursis, par rapport à L'Âge de raison, représente le "passage au grand écran ». Mais les effets de montage ne sont pas les mêmes - m'y voici donc revenu chez Malraux et chez Sartre.

Certaines scènes de L'Espoir évoquent la surimpression, et le fondu enchainé. Ainsi, devant les faces battues par la pluie de deux fuyards condamnés à mort, Manuel se souvient des visages de leurs camarades de combat, qui eux ont tenu bon «dans la même pluie, à la fin du matin ${ }^{40}$ ». À la fin de tel chapitre, il pleut sur Madrid, d'où partent des camions envoyés en renfort à Guadarrama ; le chapitre suivant s'ouvre dans la sierra de Guadarrama, « sous un grand voile de pluie oblique », tandis que l'avancée de la brigade de Manuel prend le relais du mouvement des camions ${ }^{41}$.

Il reste que jamais Malraux n'ose la superposition implicite, et dans une seule et même phrase (voire plusieurs), de différentes lignes d'action, ce que Sartre lui fait dans Le Sursis. Un seul exemple, cette phrase toute simple : «Il avait déserté et le ciel gardait son air bonhomme des dimanches ${ }^{42}$. Il suffit de la lire dans son contexte (les lignes et la page antécédentes) pour comprendre qu'elle peut aussi bien décrire les pensées de Mathieu (qui a déserté de l'Histoire et déserte la conversation avec son frère), que celles de Philippe (jeune pacifiste qui se voit en déserteur glorieux) ; aussi bien le sort de Viguier, qui ayant déserté ce monde se fait enterrer un dimanche, en libre penseur, que celui de Daniel qui au même moment dans une église défie mentalement Dieu, désertant le troupeau soumis, mais sans L'émouvoir. Dans L'Espoir ne se rencontre jamais ce procédé, qu'on a pu nommer, à propos du Sursis, pivots scripturaux (Jean-Luc Seylaz), aiguillages (Michel Contat), transits (Damien François), autant de formules qui insistent sur l'une de ses fonctions (faire passer d'une ligne d'action à une autre), mais en négligeant un peu l'autre (produire un effet de simultanéité). De sorte que je baptiserais volontiers, quant à moi, le procédé du mot de jointures, au double sens de "raccords » (Les Mots), et d'« adresse à trouver les joints, les opportunités » (Littré). Malraux ? « La syntaxe est lâche, les mots sont laids et ambigus, souvent. J'ai l'impression de relire mon premier brouillon ${ }^{43}$." Mais de cette notation cruelle (rappelons cependant que Gide avait le même avis) se tire une formule pour caractériser les jointures dans Le Sursis : il s'agit d'user de l'ambiguïté pour assurer les transitions entre les segments narratifs, et donc pour resserrer leurs liens, ou encore, en d'autres termes, pour produire ici et là une imbrication des tuiles textuelles, qui se recouvrent partiellement. Malraux, grand rival mais imparfait, donc. En revanche, le roman de Vladimir Pozner, écrivain bien moins connu, Les États-Désunis (Denoël, début 1938), a-t-il fonctionné comme un modèle caché pour Le Sursis? Oui, et l'on sait que Sartre l'avait lu - mais en tant qu'un modèle parmi d'autres : Pozner mais aussi Romains et Malraux, Dos Passos mais aussi Woolf et Joyce, le geste de Sartre consistant à totaliser un secteur de la littérature de son époque pour produire sa propre prose et sa propre forme. Curieux objet que Les États-Désunis: Pozner a séjourné aux États-Unis en 1936; il donne des extraits, datés de janvier à juin 1936, d'un «Carnet de notes »; plusieurs chapitres sont 
construits comme des enquêtes, d'autres incluent des interviews, avec des écrivains comme Dos Passos, Waldo Frank et Theodore Dreiser, ou avec des "gens de peu ", victimes du capitalisme. Donc un reportage - mais aussi un roman de montage: d'évidence le texte de Pozner praticien satisfait aux critères de Pozner théoricien, et la construction, même si jamais elle ne recourt au troublant procédé des jointures, est bien plus complexe que ce que peut tolérer la presse. Somme toute, un reportage à allure de roman. Sartre fera certes dans Le Sursis une part bien plus grande à la fiction. Mais le début de son roman doit sans doute quelque chose, selon la formule, au premier chapitre de Pozner, intitulé " Un jour comme les autres », et qui, de bref paragraphe en bref paragraphe, déplace le lecteur de lieu en lieu - les plaines du Texas, la ville de Milwaukee, un hôpital à Memphis, etc. -, tout en étant scandé par des indications horaires : $7 \mathrm{~h}$ 30, 10 heures, $10 \mathrm{~h}$ 30, 11 heures, midi, etc. Sartre d'une part précise le moment - non plus "Un jour comme les autres ", mais "Vendredi 23 septembre ", début d'une semaine de crise historique. D'autre part, il insiste sur les dissonances horaires : sur le fait que l'heure n'est pas la même à Berlin et à Londres ou Paris, par exemple, ce qui, à l'incipit du Sursis, comporte quelque valeur symbolique. Enfin, il développe les scènes, introduisant des personnages qui auront la possibilité de prendre consistance romanesque - ce qui ne se produit point dans le premier chapitre des États-Désunis (ni par la suite).

\section{Avec et par-delà le reportage}

Lucien Rebatet, qui lit Le Sursis en prison, écrit en 1946 à son ami Roland Cailleux : «très remarquable réussite technique. Procédé avalé par le mouvement d'ensemble. Bougrement du talent! Mais là, tout de même, un peu trop journalistique, reportage de grand virtuose ${ }^{44}$.» Pourtant la première page du Sursis convoque à dessein les différentes formes du temps: temps des horloges ("Seize heures trente à Berlin»), fuseaux horaires différents (« quinze heures trente à Londres »), durée vécue («L'hôtel s'ennuyait », "ils attendaient»), temps corporel ou âge ("un vieillard»), temps psychique ou mémoire ( un souvenir très ancien»), temps météorologique («une chaleur rousse ») - tout sauf l'Histoire, insaisissable, et à la hauteur de laquelle le vieil homme (Chamberlain), plus sensible au weather qu'au time, ne se trouve sans doute pas. Il y a là plus de profondeur construite, me semble-t-il, que dans un reportage même virtuose. Le Sursis est un roman à allure de reportage, mais qui dépasse et surpasse le reportage, dans son effort pour rendre « à l'événement sa brutale fraîcheur ${ }^{45}$ ".

L'allure de reportage du Sursis tient à cinq traits ${ }^{46} .1$. Le roman sélectionne un lecteur au fait de l'histoire la plus récente, qui lit le journal et donc sait, sans qu'on le lui explique, qui est Horace Wilson (évoqué à la première page) ou Sabiani (mentionné une fois en tout et pour tout). 2. Raymond Picard (le futur adversaire de Barthes), écrit en 1946 : «Le livre n'est pas distribué en chapitres », comme L'Âge de raison, « mais en journées, du 23 au 30 septembre : il y en a huit, et dans certaines, les événements s'échelonnent heure par heure. On pense au "film de la crise" dans les journaux, le lendemain des jours de tension intérieure ou extérieure ${ }^{47} »$. Non point des chapitres, donc, mais des séquences qui forment une sorte de chronique. 3. Les personnages du Sursis sont des observateurs en situation, qui n'ont pas de prise, ou presque pas, sur l'Histoire qui les déborde et les emporte. Tout comme les reporters sont d'impuissants témoins sur le vif. 4 . Le récit ne s'affranchit qu'assez rarement de la règle du point de 
vue subjectif, posée par Sartre contre Mauriac; de même qu'un reporter, quelles que soient ses prétentions à l'objectivité, ne peut éviter de sélectionner des faits et d'adopter un "angle». 5. Citant fragments de discours radiodiffusés, et titres ou couplets de chansons à la mode en 1938, le texte romanesque comporte ainsi sa bande sonore: Yefime, qui en décembre 1945 rend compte du Sursis dans Esprit, évoque le bouton de la radio que l'on tourne, passant de station en station, comme le roman de personnage en personnage.

Ce passage constitue précisément le premier moyen pour Sartre de dépasser le modèle du reportage : le fouillis simultanéiste (en fait très ordonné), c'est-à-dire la pluralité des lignes d'action qui se succèdent, ou se mêlent dans les passages tourbillons (qu'aucun article de presse, pas plus en 1938 qu'en 1945, n'a jamais donnés à lire), et qui parfois s'imbriquent (se recouvrent partiellement). Bref, le roman de montage propose un type de composition bien différent de la linéarité du reportage. Le second moyen consiste à mettre en valeur l'instant. Sartre veut montrer, dans Le Sursis, que le journalisme est incapable de saisir l'instant dans toute sa densité, qu'il soit d'ordre privé (l'instant de bonheur, ou d'angoisse) ou d'ordre public (l'instant historique). L'instant, c'est l'affaire du roman.

\section{Incanter l'instant}

«Un instant, un seul instant, il tousse, il se racle la gorge, il va chanter dans un instant » : ainsi commence, à la fin de la première séquence, un passage-tourbillon, une récapitulation partielle qui rassemble dans l'imminence tous les personnages, ou presque, évoqués jusqu'alors. Imminence de telle ou telle action ou passion privée, imminence de la guerre aussi. Instant capital, instant décisif, instant du basculement. Or l'instant ici s'installe; il se déploie sur plus de trente lignes et seulement deux phrases scandées par l'anaphore de " un instant » ou « dans un instant »; le temps qui domine est l'imparfait : il suspend la successivité, il étale une durée, il tend à faire tableau - une leçon que Sartre a retenue de Flaubert, lorsqu'il relisait L'Éducation sentimentale en décembre 1939, même si alors le procédé l'agaçait. Instant suspendu, arrêt sur instant, vertige fixé.

L'instant peut aussi être celui d'une intimité précieuse, sentie par un seul personnage, foyer de la vision. Ainsi Mathieu avec sa belle-sœur ${ }^{48}$ :

Odette lui rendit un sourire. Ce ne fut pas le sourire mondain qu'il attendait, mais un sourire pour lui tout seul; en un instant la mer fut de nouveau là, et le balancement léger de la mer et les ombres chinoises qui couraient sur les flots et la coulée de soleil qui palpitait dans la mer, et les agaves vertes et les aiguilles vertes qui tapissaient le sol et l'ombre pointilliste des grands pins et la chaleur ronde et blanche et l'odeur de résine, toute l'épaisseur d'un matin de septembre à Juan-lesPins.

La polysyndète a remplacé l'anaphore, mais on retrouve le passage du passé simple à l'imparfait, pour un tableau à la Signac (Juan-les-Pins. Soirée, 1914 ?), dans une prose poétique qui multiplie les métaphores, répète mots ou syllabes (couraient/coulaient, mer/vertes, chaleur/odeur/épaisseur); une prose, plus précisément, marquée par la poésie symboliste : les « ombres chinoises », et - avec « chaleur ronde et blanche » - la double synesthésie ${ }^{49}$. Ou enfin voici Mathieu qui dîne avec Gomez, sur une terrasse, toujours à Juan-les-Pins : 
La guerre était là, sur la piste blanche, elle était l'éclat mort du clair de lune artificiel, l'acidité fausse de la trompette bouchée et ce froid sur la nappe, dans l'odeur du vin rouge, et cette vieillesse secrète des traits de Gomez ${ }^{50}$.

On voit au mieux la manière sartrienne d'imposer par le roman la présence de l'instant, et de conférer à l'instant la présence : sur un fond initial neutre, celui du verbe être répété, que l'imparfait fige, se mettent en place rythmes binaires, échos sonores (éclat/ clair, vieillesse/secrète/traits), synesthésie encore («l'acidité de la trompette»), démonstratifs d'intensité («ce froid», «cette vieillesse »). Pour montrer comment ses personnages tiennent à l'instant, le romancier fait tenir l'instant, comme une note qui se prolonge.

Certes, si Mathieu se montre à ce point sensible à un instant, c'est qu'il se trouve encore dans la contemplation: l'heure de l'action n'a pas sonné pour lui. On peut pourtant apprécier avec lui cette "poésie de l'éphémère ", cette "euphorie de la finitude ${ }^{51}$ ". Même dans l'angoisse ; tel est le sentiment d'Ivich arrivant à Paris et dévorant des yeux la ville : «Même s'ils lâchaient leurs bombes dans cinq minutes, dans trente secondes, ils ne pourraient pas m'enlever ça ${ }^{52}$. » Rien de plus simple que ce démonstratif, que ne soulignent même pas des italiques. Il porte pourtant en lui toute la présence de cet instant. Pour la faire sentir, on aura compris que Sartre joue de toute une gamme de procédés, qui vont de ce simple pronom jusqu'au « style sorcier » dont Beauvoir parlait à Jacques-Laurent Bost ${ }^{53}$, style qui incante l'instant : incarne dans les mots tel état du monde à tel instant, avec tout son prix. Opération qui s'appuie à la fois sur un appel à la connivence du lecteur, lequel partage avec l'auteur une certaine expérience du monde ("cette vieillesse secrète »), et sur le mimologisme magique profondément ancré en Sartre depuis son enfance (voir Les Mots).

Or, évidemment, le journaliste, lui, n'est ni un poète ni un sorcier ; disons même qu'il serait malvenu à faire le poète ou le sorcier dans un reportage ; informer, en principe, n'est pas envoûter. Pourtant, le journalisme élève lui aussi des prétentions à saisir l'instant en cours, ou au plus près de sa course. Daniel Halévy, bien qu'il rédige au passé simple, au passé historique, se présente comme " travaillant [...] sur l'instantané ${ }^{4}$ ». On songe aussitôt à la formule de Camus, qui dans Combat, le $1^{\mathrm{er}}$ septembre 1944, définira le journaliste comme « un historien au jour le jour ${ }^{55}$ " - formule souvent devenue : un « historien de l'instant » (et qui fit débat). À preuve encore, dans Le Sursis, l'expression de « Dernière heure ", pour caractériser (nous sommes, dans la diégèse, en 1938) le plus récent tirage de l'édition d'un journal, actualisée, mise à jour.

\section{La dernière heure}

Prétentions dont en fait Sartre s'amuse. Ainsi voit-on Maurice Gounod acheter « ParisSoir, dernière » : mais il est déçu, «c'est toujours pareil ${ }^{56}$ ». Rien de neuf du côté des événements, aussi la dernière répète-t-elle l'avant-dernière. Autre déception possible, un événement négatif - celui que découvre une infirmière, dépliant le journal: «Dernière heure. M. Chamberlain n'a pas conféré, cet après-midi, avec le chancelier Hitler $^{57}$. » Or cette infirmière est en train de veiller le corps d'Armand Viguier, tout fraîchement décédé. La manchette "Dernière heure" prend alors une valeur rétroactive : elle amène à relire autrement les lignes qui précédent.

C'est pour Viguier que la véritable dernière heure a sonné. Et au vide du journal s'oppose la plénitude des lignes qui viennent de décrire sa vie à l'instant où la mort la 
résume, du côté de Thanatos puis d'Éros : « les cris étaient là, les rires, le sifflement des locomotives et l'éclatement des schrapnells, le 6 mai 1917, ce bombardement sanglant dans sa tête, quand il tombe entre les deux tranchées ", mais aussi «ce grand soleil immobile qui éclaire pour toujours un visage de femme, à la Grande Jatte, le 10 juillet 1900 , dans le canot ${ }^{58} »$. On retrouve la manière déjà repérée de poser l'instant, de l'imposer, de le faire peser : un début sur un mode mineur, qui établit platement une existence (" étaient là »), puis la phrase prend son élan et sa densité, avec les effets sonores (cris/rires, éclatement/sifflement/bombardement, tête/tombe/tranchées), le démonstratif d'intensité, le glissement de l'imparfait au présent (" quand il tombe »). L'instant vécu devient éternel (« pour toujours »), le relatif se fait absolu.

Relevons aussi le paradoxe que suggèrent les déterminants de notoriété : «les deux tranchées ", « le canot » (et non pas un canot). Objets, réalités, bien connus de Viguier, auxquels il a souvent pensé. Qui peut ainsi pénétrer dans l'esprit d'un mort? Non pas un journaliste. Mais un romancier usant - c'est bien le moment d'y recourir pour cette nécromancie - d'un style sorcier. Au prix d'une entorse à sa propre règle du point de vue, anti-mauriacienne: Sartre ici se fait Asmodée, il soulève non pas le toit des maisons mais le couvercle d'un crâne. Et qu'est-ce qui l'autorise à ce coup de force, outre le célèbre droit de se contredire revendiqué par Baudelaire? Un accès de bergsonisme: dans son article de 1938 sur Dos Passos et 1919, Sartre évoquait «ces mémoires bergsoniennes qui flottent, après la mort du corps, pleines de cris et d'odeurs et de lumières, et sans vie, dans on ne sait quelles limbes ${ }^{59}$ ». Curieuse adoption d'une théorie spiritualiste de la mémoire, par un romancier qui avait écrit dans La Nausée (ce roman assez largement anti-proustien) que "l'existence est sans mémoire: des disparus, elle ne garde rien - pas même un souvenir ", et par un philosophe athée, qui du passé propose une analyse bien différente dans L'Être et le Néant.

Il arrive aussi que le récit épouse le point de vue non pas d'un mort mais d'un mourant - d'un homme sur le point de vivre sa dernière heure, même s'il l'ignore. Dans la réalité (mise en place par la diégèse), ainsi du Marocain franquiste lors des combats de la Cité Universitaire à Madrid :

[...] il avait peur, il pensait à Tanger ; tout en haut de Tanger il y avait une maison jaune à un étage d'où l'on voyait le scintillement éternel de la mer. [...] Il fallait penser à cette maison jaune $[. . .]^{60}$.

Ou bien en imagination, et voici Pierre, victime à la fois du mal de mer et de sa peur :

Onze heures; le ciel fourmillait d'étoiles, un feu rouge dansait au loin sur la mer; c'est peut-être cette image-là qui reviendra dans mes yeux la dernière et qui s'y fixera pour toujours, quand je serai dans mon entonnoir, à la renverse avec la mâchoire emportée, sous un ciel clignotant. Cette pure image noire avec ce bruissement de palmes et ces présences d'hommes, si lointaines derrière leur feu rouge, dans le noir ${ }^{61}$.

Un décor aux couleurs infernales, noir et rouge; un noir d'angoisse qui se diffuse phoniquement (étoiles/entonnoir/mâchoire/noire/dans le noir); une image d'abord vue par Pierre, dans un récit à l'imparfait, puis qu'il projette vers le futur, et enfin vécue au présent: le discours direct libre tourne alors au monologue intérieur, avec phrase nominale (donc plus de repérage temporel), et démonstratifs de la présence intense. Tout conduit ce pauvre Pierre vers l'hypotypose, et une danse de morts, mais au ralenti, surgit sous ses yeux :

Il les vit, en uniforme, serrés comme des harengs derrière leur fanal, glissant silencieusement vers la mort. Ils le regardaient sans souffler mot, le feu rouge glissait sur l'eau, ils glissaient, ils défilaient devant Pierre et ils le regardaient ${ }^{62}$. 
Selon Les Mots, la grand-mère de Sartre, Louise Schweitzer aimait à dire : "Glissez, mortels, n'appuyez pas.» Ici les mortels glissent encore, une fois morts, en une procession funèbre.

41 La généralisation va de soi, et elle se lit dans un compte rendu en 1945 : «Le Sursis est un livre où le lecteur est obligé de rentrer dans la danse », une "danse de moribonds affolés par la vision d'un monde qui bascule ", la danse de la dernière heure, ou du dernier instant, celle où l'on voit défiler sa vie sur son lit de mort, avec une «tension passionnée ${ }^{63}$ ». C'est ce qui fait pour une bonne part la réussite du roman. Sartre écrivait de Dos Passos qu'il s'était « installé, dès les premières lignes de son livre, dans la mort ${ }^{64} »$. Curieuse posture, celle de 1919, mais aussi du Sursis. Le bénéfice pour le roman est évident, si elle favorise "l'un des fondements du romanesque qui est la nostalgie $e^{65}$ ».

Le roman sartrien comme vaste épiphanie de la dernière heure, des derniers instants, du dernier instant, dans la nostalgie de la vie perdue ou sur le point de l'être? Problème : dès le 18 février 1940, alors qu'il n'a pas encore commencé à écrire Le Sursis, Sartre se reproche déjà d'avoir «fait sans y prendre garde une philosophie de l'instant ${ }^{66} "$, assez apparente en effet dans La Nausée. Il la délaisse formellement dans L'Être et le Néant: "Il est impossible de saisir le présent sous forme d'instant car l'instant serait le moment où le présent est. Or le présent n'est pas, il se présentifie sous forme de fuite ${ }^{67} »$. Sartre romancier tente donc dans Le Sursis une saisie et une poésie de l'instant (souvent une poésie-pour-saisir) - que Sartre philosophe n'assume déjà plus. Et qu'il ne reprendra plus jamais à son compte ${ }^{68}$.

Pourquoi ce décalage?

Il a d'abord le mérite de nous rappeler que Sartre romancier n'est pas, quoi qu'on en dise trop souvent, le simple illustrateur des thèses de Sartre philosophe. Ici, plutôt, son contradicteur. De façon générale, sans doute, son inquiéteur - pour reprendre un mot cher à Villiers de l'Isle-Adam (Histoires insolites), puis à Gide. Peut-être Sartre s'en remet-il au roman pour méditer une question que le philosophe en lui ne sait pas encore penser ou ne veut pas encore penser (l'articulation de l'instant et de la dialectique dans l'Histoire, les différents types de sens ou de non-sens qu'on y rencontre).

D'autre part, le 9 mars 1940, Sartre note : "J'enrage de n'être pas poète ${ }^{69}$ ». Sur un strict plan littéraire, la valorisation de l'instant lui permet de créer ici et là, dans la prose du Sursis, de fugaces poèmes, qui sont, écrit-il dans la même page du carnet XIV, « comme l'éternité d'un instant ». Comme l'inscription, donc, de l'absolu dans le relatif - tout ce que le journalisme, décidément, faute de sens poétique, échouerait à accomplir.

Enfin, sur un plan philosophique, valoriser l'instant permet à Sartre d'échapper au relativisme historique. C'est-à-dire à la thèse qui lui semble celle d'Aron dans son Introduction à la philosophie de l'histoire, un ouvrage qu'il a lu de près dès sa parution en 1938. Aron a dit à Sartre que son Introduction à la philosophie de l'histoire était un "plaidoyer pour l'athéisme philosophique et méthodologique ${ }^{70}$ ». En effet, pour lui, note Sartre, l'événement historique «en soi, c'est l'événement tel qu'il apparaîtrait à Dieu ». Or l'historien n'est pas Dieu; il est lui-même non pas éternel mais historique. Donc jamais il ne saisit l'événement historique comme il fut, mais sur le mode du relatif (à lui, à son époque, à ses méthodes, à ses préjugés, à ses intérêts). Sartre voudrait bien entendu ne pas recourir à Dieu, mais ne pas non plus tomber dans le relativisme. Il lui 
faut donc mettre l'absolu dans le relatif même. C'est-à-dire créer un absolu vécu par le sujet humain.

À l'époque du Sursis, cet absolu, Sartre le place dans l'instant vécu sous la menace de la mort. Cela veut dire aussi : dans l'historicité, dans la conscience vécue de l'Histoire en train de se faire. Peut-être la possibilité se dessine-t-elle alors de penser non plus en termes d'instants, mais d'époque - puis de dialectique de l'Histoire dans l'époque. Sartre y viendra ${ }^{71}$. Mais il n'en est pas encore là. Il use du roman pour tenter - entre autres objectifs, bien sûr -, plaisir dont il ne se lasse pas depuis l'École normale, de « réfuter Aron ${ }^{72}$ ». Aussi le roman de l'imminence (de la guerre) devient-il roman de l'immanence (de l'absolu au relatif).

L'amusant est que Sartre va retourner contre Aron certains de ses thèmes et même de ses tours de plume. De ses thèmes : «si on néglige les décisions et les instants, écrit Aron, on substitue au monde vécu une nature ou une fatalité ${ }^{73}$ »; et encore: «Pour rendre après coup à l'événement sa qualité propre, il faut, en l'opposant aux nécessités partielles, marquer l'action de l'individu ou de l'instant ${ }^{74} »-$ contre toute forme de déterminisme, la liberté sartrienne a, elle aussi, partie liée avec l'instant, la décision ou le choix de l'individu. De ses tours, puisque Aron déjà associe le déterminant démonstratif et le concret absolu ou l'événement pur ${ }^{75}$.

L'amusant de l'amusant est que si Sartre pille quelque peu Aron, qu'il ne cesse pas pour autant de critiquer, Claude Simon lui réservera le même sort. Qu'on relise ce qu'il disait en 1987, à propos de La Route des Flandres, cet autre roman de montage : «ce n'est pas une histoire dans l'acception traditionnelle du mot lorsqu'on parle de roman : c'est la tentative de description de tout ce qui peut se passer en un instant en fait de souvenirs, d'images et d'associations dans un esprit ${ }^{76}$ » - autour d'une « image-mère » qui revient à la mémoire. Mots qui ne s'appliquent pas mal au Sursis, si l'on remplace un esprit par une multiplicité de consciences. Le gonflement monstrueux de l'instant presque ultime, et donc à jamais traumatique: la mort d'un colonel (dans la réalité vécue) ou d'un capitaine (dans le roman) abattu en mai 1940 sur une route des Flandres, la guerre sur le point de déferler en septembre 1938, voilà ce que Sartre et Claude Simon, deux soldats qui n'ont jamais tiré un coup de fusil, deux prisonniers de guerre, et peut-être prisonniers de la pensée de la guerre, ont écrit - chacun à sa manière, certes, manière non pas de reporters de guerre, mais de romanciers.

\section{NOTES}

1. J.-P. Sartre, Euvres romanesques, M. Contat et M. Rybalka (éd.), Paris, Gallimard, coll. «Bibliothèque de la Pléiade », 1981, p. 1970.

2. Continuer : voir J.-F. Louette, «Le Sursis : petite critique de la raison journalistique », Sartre et Beauvoir, roman et philosophie, Genève, La Baconnière, 2019, p. 121-143.

3. D. Bougnoux, "L'œuvre et le flot: du journal au roman ", Recherches et travaux, Journaux et journalistes. Hommage à Jean Sgard, $\mathrm{n}^{\circ}$ 48, 1995, p. 171.

4. J.-P. Sartre, Lettres au Castor et à quelques autres, Paris, Gallimard, 1983, t. I, p. 215. 
5. Ibid., t. I, p. 518.

6. Ibid., t. II, p. 11.

7. Ibid., t. 1, p. 420.

8. Ibid., t. I, p. 423.

9. Ibid., t. I, p. 271 (31 août 1939).

10. S. de Beauvoir, Lettres à Sartre, Paris, Gallimard, 1990, t. I, p. 356.

11. Ibid., t. I, p. 367 (20 décembre 1939).

12. Ibid., t. I, p. 392.

13. A. Fabre-Luce, Histoire secrète de la conciliation de Munich, Paris, Grasset, 1938, p. 11 et 66.

14. Ibid., p. 90, 91, 97.

15. J.-P. Sartre, Lettres au Castor et à quelques autres, t. II, ouvr. cité, p. 248. L'autre Fabre-Luce est le baron Robert Fabre-Luce : Sartre sait sans doute que dans les années vingt il avait voulu lancer en Europe un parti populaire chrétien de la nouvelle droite, et que, favorable à l'Allemagne nazie, il avait été en 1933 l'un des fondateurs de l'Alliance raciste européenne.

16. Voir J.-F. Louette, Sartre et Beauvoir, roman et philosophie, ouvr. cité.

17. P. Nizan, Chronique de septembre, Paris, Gallimard, 1939, p. 7.

18. J.-P. Sartre, Carnets de la drôle de guerre, "Les Mots» et autres écrits autobiographiques, J.F. Louette (éd.), Paris, Gallimard, coll. « Bibliothèque de la Pléiade », 2010, p. 599.

19. P. Nizan, Chronique de septembre, ouvr. cité, p. 15 et 8.

20. J.-J. Brochier, Paul Nizan intellectuel communiste, Paris, Maspero, 1970, t. I, p. 118.

21. A. Vaillant, L'Histoire littéraire, Paris, Armand Colin, 2010, p. 350.

22. B. Frank, Les Rats [1953], Paris, Flammarion, coll. «Le Livre de Poche», 1985, p. 441. La conversation de Bourrieu avec Sartre, dans le roman, se passe en 1951 ou 1952. Sartre a fait l'éloge du reportage à la fin du «Manifeste » des Temps modernes ( ${ }^{\circ} 1$, octobre 1945), mais la formule attribuée à Malraux résume de façon quelque peu simplificatrice sa Préface au livre d'Andrée Viollis, Indochine S.O.S., Paris, Gallimard, coll. « Les Documents bleus », 1935.

23. V. Chklovski, Voyage sentimental, Paris, Simon Kra, 1926, p. 218.

24. Voir M. Boucharenc, L'Écrivain-reporter au cœur des années trente, Villeneuve-d'Ascq, Presses universitaires du Septentrion, 2004.

25. Publié aux États-Unis en 1925; adapté au cinéma, sous le même titre, par Joseph von Sternberg en 1931 ; traduit en deux volumes chez Fayard au début de 1932 (a. i. du 17 mars), par Victor Llona.

26. M. Autrand, «Introduction», A. Malraux, Euvres complètes, Paris, Gallimard, coll. «Bibliothèque de la Pléiade », 1996, t. II, p. XVIII.

27. J.-P. Sartre, Le Sursis, Paris, Gallimard, coll. « Folio », 2009, p. 290 et p. 303.

28. Voir J.-P. Sartre, Le Sursis, ouvr. cité, p. 290, p. 294-295 (le point de vue du rebelle), et p. 298-299 (sa mort).

29. Ibid., p. 369.

30. J.-P. Sartre, Lettres au Castor, ouvr. cité, t. I, p. 434.

31. Lettre à Beauvoir du 28 avril 1940 (Lettres au Castor, t. II, ouvr. cité, p. 196).

32. H. Thomas, « De l'intime à l'épique », Terre des hommes, $n^{\circ}$ 4, 20 octobre 1945, p. 9.

33. B. Frank, Le Dernier des Mohicans [1956], Paris, Grasset, coll. «Cahiers rouges», 2011, p. $109-110$.

34. J.-P. Sartre, Carnets de la drôle de guerre, ouvr. cité, p. 578-579.

35. Voir les précieux travaux de Jean-Pierre Morel, notamment «Montage, collage et discours romanesque dans les années vingt et trente ", dans Denis Bablet (éd.) Collage et montage au théâtre et dans les autres arts, Lausanne, L'Âge d'homme, 1978, p. 38-73 ; " "Carrefour multiple" : roman et montage dans Le Sursis », Dix-neuf/Vingt, $\mathrm{n}^{\circ} 10$, octobre 2000, p.191-208, repris dans J.F. Louette (éd.), Sartre écrivain, Paris, Eurédit, 2005, p. 101-123; et «Cinq difficultés - au moins - 
pour parler de montage en littérature ", dans Robert Kahn (éd.), À travers les modes, Rouen, Publications de l'Université de Rouen, 2004, p. 35-48.

36. V. Pozner, Panorama de la littérature russe contemporaine, Paris, Kra, 1929, p. 362-363.

37. A. Malraux, Essais, Jean-Yves Tadié (éd.), Euvres complètes, Paris, Gallimard, coll. «Bibliothèque de la Pléiade », t. VI, 2010, p. 296 et 297. Les Traqués a paru chez Gallimard le 8 décembre 1933.

38. V. Pozner, Panorama de la littérature russe contemporaine, ouvr. cité, p. 363.

39. "[...] nous lisions tous les jeunes auteurs russes qui étaient traduits en français" (S. de Beauvoir, Mémoires, J.-L. Jeannelle et É. Lecarme-Tabone (éd.), Paris, Gallimard, coll. «Bibliothèque de la Pléiade », 2018, p. 394). Beauvoir cite Nous autres de Zamiatine (1929) et Rapace d'Ehrenbourg (1930), parus dans la collection «Les Jeunes Russes », mais aussi La Volga se jette dans la Caspienne (Paris, Éditions du Carrefour, février 1931).

40. A. Malraux, L'Espoir, Euvres complètes, t. II, ouvr. cité, p. 332.

41. Ibid., p. 301 et 302.

42. J.-P. Sartre, Le Sursis, ouvr. cité, p. 237.

43. J.-P. Sartre, Carnets de la drôle de guerre, 27 mars 1940, ouvr. cité, p. 648-649.

44. L. Rebatet, Lettres de prison. 1945-1952, Paris, Le Dilettante, 1993, p. 120.

45. J.-P. Sartre, Qu'est-ce que la littérature ?, Situations, II [1948], Paris, Gallimard, 1978, p. 254. Voir B. Denis, «"Rendre à l'événement sa brutale fraîcheur". Roman et événement chez JeanPaul Sartre ", Que se passe-t-il ? Événement, sciences humaines et littérature, D. Alexandre (éd.) et al., Rennes, Presses universitaires de Rennes, 2004, p. 213-222.

46. Voir notre Sartre et Beauvoir, roman et philosophie, ouvr. cité.

47. "L'Art de Jean-Paul Sartre et les "Hommes de mauvaise volonté" ", La France libre, n 64, 15 février 1946. Né en 1917, Raymond Picard vient en 1945 d'obtenir l'agrégation de lettres.

48. J.-P. Sartre, Le Sursis, ouvr. cité, p. 124.

49. «En un sens, assurément, J.-P. Sartre est symboliste, et du reste, ce sont des procédés symbolistes qu'il emploie ; citons le plus courant, la "correspondance", qui consiste à transposer une sensation d'un clavier sensoriel à un autre. [...] J.-P. Sartre manie les mots en poète symboliste, qui sait peser leur valeur musicale et leur puissance d'évocation [...] » (Raymond Picard, art. cité, p. 292).

50. J.-P. Sartre, Le Sursis, ouvr. cité, p. 315.

51. G. Idt, «L'engagement dans Journal de guerre I, de Sartre », Revue philosophique de la France et de l'étranger, t. CXX, nº 2, juillet-septembre 1996, p. 402.

52. J.-P. Sartre, Le Sursis, ouvr. cité, p. 438.

53. S. de Beauvoir, J.-L. Bost, Correspondance croisée 1937-1940, Paris, Gallimard, 2004, p. 296 (lettre du 27 mars 1939).

54. D. Halévy, 1938, une année d'histoire, Paris, Grasset, 1938, p. 31.

55. A. Camus, "La réforme de la presse », dans Jacqueline Lévi-Valensi (éd.), CEuvres complètes, Paris, Gallimard, coll. « Bibliothèque de la Pléiade », 2006, t. II, p. 521.

56. J.-P. Sartre, Le Sursis, ouvr. cité, p. 9.

57. Ibid., p. 71.

58. Ibid., p.70. On pense au tableau célèbre de Seurat: Un dimanche après-midi à l'île de la Grande Jatte, 1886.

59. Situations, I [1947], Paris, Gallimard, 1978, p. 18. Thèse récurrente, avec quelques inflexions, chez Bergson, de Matière et mémoire (1896) aux Deux Sources de la morale et de la religion (1932, fin du ch. III), en passant par la conférence sur « La perception du mouvement » (1911) reprise dans La Pensée et le Mouvant (1938).

60. J.-P. Sartre, Le Sursis, ouvr. cité, p. 290.

61. Ibid., p. 201.

62. Ibid., p. 201-202. 
63. P. Morelle, Volontés de ceux de la Résistance, 31 octobre 1945, p. 3.

64. « À propos de John Dos Passos et de 1919 », art. cité, p. 18.

65. B. Frank, Le Dernier des Mohicans, ouvr. cité, p. 104.

66. J.-P. Sartre, Carnets de la drôle de guerre, ouvr. cité, p. 436.

67. J.-P. Sartre, L'Être et le Néant [1943], Paris, Gallimard, coll. «Bibliothèque des idées », 1971, p. 159.

68. Car l'instant, certes "image de l'éternité » dans l'éphémère, serait "négation du temps humain, ce temps à trois dimensions du travail et de l'histoire " (Qu'est-ce que la littérature?, ouvr. cité, p. 173). Et dans L'Idiot de la famille : l'hédonisme n'est qu'un « rêve bleu. Pour y croire, il fallait s'imaginer qu'une personne tout entière, avec toutes ses ressources et toutes ses passions, pût se résumer dans le plaisir d'un instant. Impossible : l'homme est futur " (Paris, Gallimard, coll. « Bibliothèque de philosophie », 1971, t. II, p. 1729).

69. J.-P. Sartre, Carnets de la drôle de guerre, ouvr. cité, p. 564.

70. Ibid., p. 490.

71. Deux textes majeurs sur ce point, l'un anthume et l'autre posthume : « [...] au plus profond de la relativité historique et de notre insignifiance, nous sommes des absolus, inimitables, incomparables, et notre choix de nous-mêmes est un absolu » («Écrire pour son époque » [1946], Les Temps modernes, juin 1948, p. 2115, repris dans Situations II, Paris, Gallimard, 2012) ; et en 1947-1948: « [...] l'Histoire n'a pas de dehors d'où l'on puisse la juger ou faire la somme de ce qu'elle fut. L'Histoire et les événements historiques ne présentent jamais de sens absolu qui serait transcendant au relatif, mais bien au contraire chaque conjoncture historique est relative et l'absolu est immanent au relatif. L'absolu ce n'est pas le point de vue de Dieu sur l'Histoire, c'est la façon dont chaque homme et chaque collectivité concrète vit son histoire. En renonçant à l'absolu transcendant vous ne tombez pas dans le relativisme, vous rendez à l'homme sa valeur absolue » (J.-P. Sartre, Cahiers pour une morale, Paris, Gallimard, coll. «Bibliothèque de philosophie », 1983, p. 437).

72. Sartre à Beauvoir, 12 mars 1940 : «j'ai écrit dans le carnet sur l'histoire ; tout va par problème dans le carnet; depuis huit jours c'est l'histoire, et de réfuter Aron, naturellement " (Lettres au Castor, ouvr. cité, t. II, p. 132).

73. R. Aron, Introduction à la philosophie de l'histoire, Paris, Gallimard, 1938, p. 187.

74. Ibid., p. 261.

75. Ibid., p. 19: «Plus nous nous rapprocherons du concret absolu, de cette chute localisée, décrite exactement [...]»: et p. 42 : «L'événement est le donné le plus primitif : ce qui n'est pas mais se passe, traverse l'existence à la limite insaisissable qui sépare deux instants ; cette pierre est tombée, cet individu a poussé un cri ».

76. L. Dällenbach, Claude Simon, Paris, Le Seuil, coll. «Les contemporains », 1988, p. 178-179 et 181.

\section{RÉSUMÉS}

Comment raconter au mieux la dernière heure? Question que Sartre se pose dans son roman Le Sursis (1945), qui relate la crise de Munich (septembre 1938). Réponse : ne pas s'en remettre à la presse, ni à l'histoire immédiate (Fabre-Luce). Mais oser le roman d'actualité, à faible distance romanesque des événements, dont Nizan et Chamson ont donné de tout récents exemples. Ou 
Malraux : mais Sartre, sur le même terrain - roman à allure de reportage, roman de montage prend ses distances: il invente les aiguillages ou jointures, entre phrases et entre scènes, que l'auteur de L'Espoir ne pratique pas ; il cherche à incanter l'instant: de l'amour et de l'angoisse, du trépas et de l'Histoire en suspens. L'enjeu? Se faire, fugacement, poète ; et échapper au relativisme historique : dans l'instant même résiderait un absolu.

How best to tell the last hour? A question that Sartre asks himself in his novel The Reprieve (1945 in French, 1947 in English), which relates the Munich crisis (September 1938). Answer: don't rely on the press, nor on immediate history (Fabre-Luce). But dare to write a novel about current events, at a short romantic distance from the events, of which Nizan and Chamson have given very recent examples. Or Malraux: but Sartre, on the same ground-a novel with the allure of a reportage, a montage novel-takes his distance: he invents switches or joints, between sentences and between scenes, which the author of Mans Hope does not practice; he seeks to incant the moment: of love and anguish, of death and History in suspense. What is at stake? To become, fleetingly, a poet; and to escape from historical relativism: in the very instant would reside an absolute.

\section{INDEX}

Mots-clés : poésie, roman, littérature, journalisme, Sartre, Malraux

Keywords : poetry, novel, literature, journalism, Sarte, Malraux

\section{AUTEUR}

\section{JEAN-FRANÇOIS LOUETTE}

Jean-François Louette, professeur à la Sorbonne depuis 2005, a publié plusieurs ouvrages sur Sartre, dont il a notamment édité, pour la Bibliothèque de la Pléiade, "Les Mots » et autres écrits autobiographiques (Gallimard, 2010). C'est en travaillant sur Le Sursis (Les Chemins de la liberté, t. II) qu'il s'est intéressé aux problèmes du reportage. 